\title{
Selection of depression measures for use among Vietnamese populations in primary care settings: a scoping review
}

\author{
Jill Murphy ${ }^{1 *}$, Elliot M. Goldner ${ }^{1}$, Charles H. Goldsmith ${ }^{1,2}$, Pham Thi Oanh ${ }^{3}$, William Zhu ${ }^{4}$, Kitty K. Corbett ${ }^{5}$ \\ and Vu Cong Nguyen ${ }^{3}$
}

\begin{abstract}
Depression is an important and growing contributor to the burden of disease around the world and evidence suggests the experience of depression varies cross-culturally. Efforts to improve the integration of services for depression in primary care are increasing globally, meaning that culturally valid measures that are acceptable for use in primary care settings are needed. We conducted a scoping review of 27 studies that validated or used 10 measures of depression in Vietnamese populations. We reviewed the validity of the instruments as reported in the studies and qualitatively assessed cultural validity and acceptability for use in primary care. We found much variation in the methods used to validate the measures, with an emphasis on criterion validity and reliability. Enhanced evaluation of content and construct validity is needed to ensure validity within diverse cultural contexts such as Vietnam. For effective use in primary care, measures must be further evaluated for their brevity and ease of use. To identify appropriate measures for use in primary care in diverse populations, assessment must balance standard validity testing with enhanced testing for appropriateness in terms of culture, language, and gender and for acceptability for use in primary care.
\end{abstract}

Keywords: Vietnam, Depression, Measures, Primary care, Culture

\section{Background}

While depression has been cited as a prominent and growing contributor to the global burden of disease [1], the construct and experience of depression may differ cross-culturally, with extensive variation in the expression of emotions, prominence of somatic symptoms and experience of and coping with suffering [2]. Despite this variation, evidence suggests that "depressive states can be studied as a feature of local forms of suffering" across the globe [2] and that symptoms associated with depression are present in all cultures [3]. The universality of depressive conditions combined with cross-cultural variation in the experience of depression mean that their identification and treatment are not culturally neutral. Strategies and tools that are culturally valid are essential.

\footnotetext{
*Correspondence: jgmurphy@sfu.ca

${ }^{1}$ Faculty of Health Sciences, Simon Fraser University, Blusson Hall, Room 11300, 8888 University Drive, Burnaby, BC V5A 1S6, Canada Full list of author information is available at the end of the article
}

The integration of mental health services into primary care is a recommended approach to address a large and growing gap in treatment $[4,5]$. In the time-and resource-constrained context of primary care, identifying appropriate measures is needed to improve the use of limited resources and access to care [6].

Vietnam is a lower middle-income country with a diverse population of approximately 90 million people [7] and 54 distinct ethnic groups [8]. Over 4 million Vietnamese also live abroad [9]. Mental health services in Vietnam have been largely concentrated in tertiary psychiatric facilities and have focused on schizophrenia and epilepsy [10-12]. The availability of trained mental health practitioners in Vietnam is very low, with approximately 1 per 100,000 persons [13]. Primary health care service delivery is provided by 10,750 commune health stations that operate on a catchment system and staff approximately 47,000 primary care providers throughout the country [13]. The nature and scope of services and capacity in the primary care sector varies throughout 
the country. The government of Vietnam launched the National Community Mental Health Care (CMHC) project in 2001, with the goal of integrating mental health services into primary care. In practice, the CMHC emphasizes the provision of medications for patients with mental disorders although delivery and resources vary throughout the country [13]. Although depressive disorders are prevalent in Vietnam [11, 14, 15] evidence about their treatment is limited, suggesting that little has been done to build treatment capacity, especially at the primary care level. The government has recently shown interest in improving and expanding community-based services for depression [16].

This scoping review examines measures of depression that have been used in Vietnam or among the Vietnamese diaspora. The review assesses the validity of the measures as reported in the literature, taking into account the whether their cultural validity has been tested, the context in which they were validated or used, and their appropriateness for use in primary care with the objectives of: (1) making recommendations regarding the use of depression measures in primary care with Vietnamese populations and cross-culturally; (2) identifying gaps in the existing evidence.

\section{Review}

Scoping reviews are appropriate to map "the relevant literature in the field of interest", allowing for findings to be summarized and gaps identified [17]. Scoping reviews are rigorous, with methods that allow for replication, but findings are not synthesized or aggregated to the extent customary in systematic reviews. They allow for the inclusion of diverse study designs and involve an iterative search process where search terms may evolve during the review [17]. This method allows for identifying a broad range of studies.

We searched PubMed, PsychInfo and IDRC databases on June 12th and June 16th 2014. Search terms included: depression, mental disorder and mental health; scales, measures and screening; Vietnam and Vietnamese; we excluded 'veterans' given the many studies on American veterans [18]. Studies were included that validated or used measures deemed appropriate for use in general populations. The review included studies that explicitly validated instruments and those that used instruments in research, as several studies did not validate the instruments but nonetheless produced findings relevant to this review. Given the limited volume of research in this area, the search was not limited by date of publication.

Figure 1 shows the search and selection processes undertaken in this review. The database search identified 91 articles, which was reduced to 78 after removing duplicates. Initial screening eliminated 6 additional titles.
Seventy-two full-text articles were reviewed for eligibility, with 44 then excluded. Studies were excluded because they: used data from the Vietnam Era Twin Study and were not related to Vietnamese populations; focused on neurological disorders or genetic markers; focused on specific rather than general populations or measures (e.g. scales to measure post-partum depression); or were not related to depression. Twenty-eight articles were first included in the review, with 11 measures initially identified. One measure and its source paper were excluded post hoc, since it compared mental health status in immigrants in Europe without discussing considerations specific to the measure or its use in Vietnamese populations [19]. The final set was 27 papers, with 10 measures identified.

Validation of psychometric measures is a broad methodological domain, with validity referring to "how well one can legitimately trust the results of a test as interpreted for a specific purpose" [20] or as "the ability of the instrument to measure the attributes of the construct under study" [21]. Validity typically addresses construct, content and criterion validity, and measures of reliability [20, 21]. Considerations such as semantic equivalence, which examines linguistic and idiomatic equivalence [22, 23], may also be included. Construct validity is the "degree to which an instrument measures the construct it is intended to measure" [21]. Content validity refers to whether the items in a measure reflect the full range of characteristics of the construct being examined. Criterion validity examines how a measure performs in relationship to another instrument or test. Finally, reliability examines the consistency with which a measure accurately identifies the construct of interest. Reliability is necessary but not sufficient for validity [20, 21]. Cook and Beckman [20] recommend that conclusions about an instrument's validity should be based on several data types. The studies we reviewed employed various methods, some more comprehensive than others, to draw conclusions about validity.

Socio-cultural context is an essential consideration when assessing and understanding depression. This review includes measures that were developed for use in Western countries and subsequently applied with Vietnamese populations. We consider both the studies' statistical results and qualitative observations about the appropriateness and cross-cultural validity of the measures in the context of the populations studied. We also consider factors influencing the appropriateness of measures for use in primary care. While the included studies did not all take place in primary care settings, characteristics of the measures, including the instrument's length and accessibility, were identified as relevant to the measures' appropriateness for use in such 

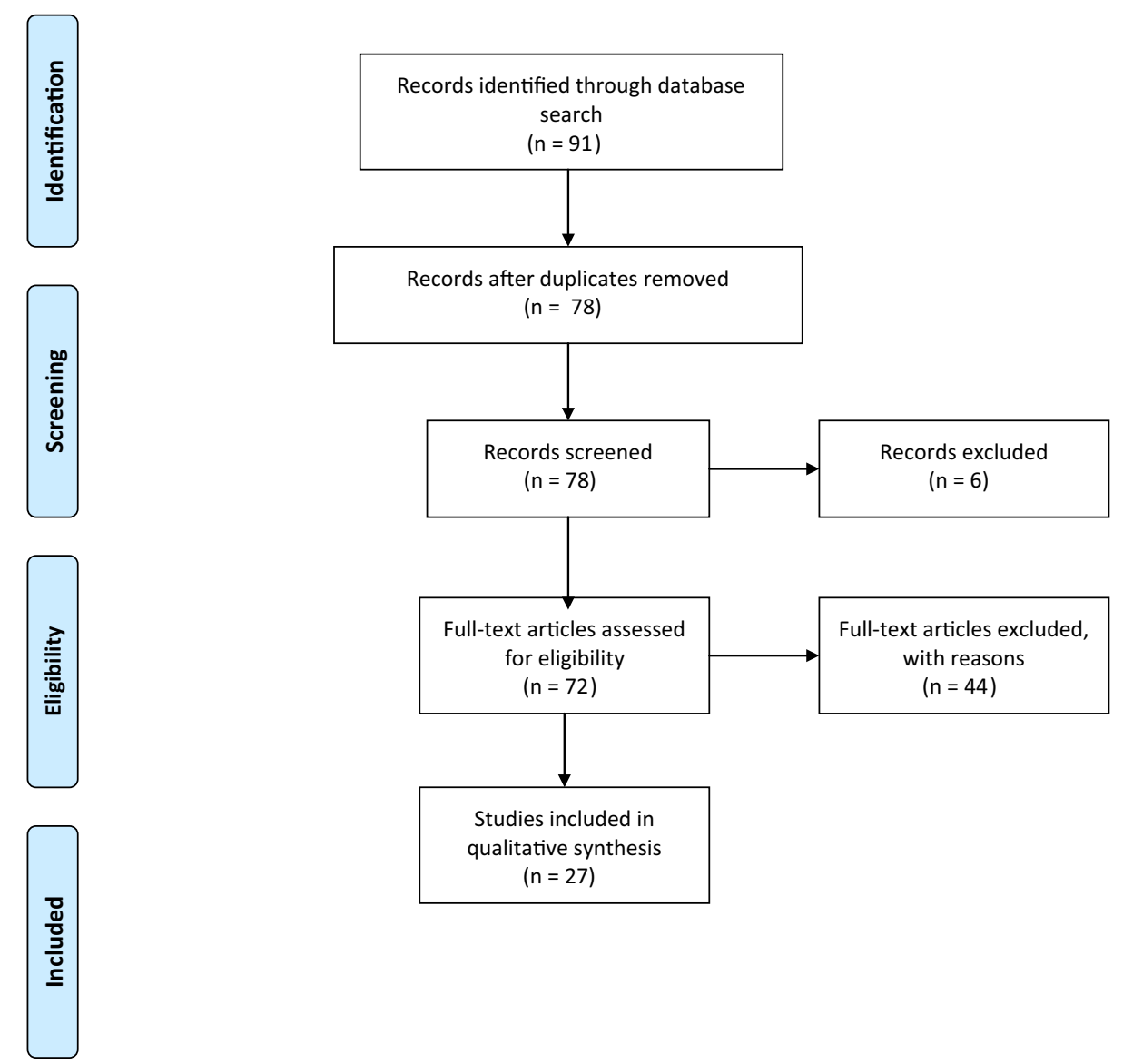

Fig. 1 PRISMA diagram: search and selection process using Pubmed, PsychInfo and IDRC databases

settings. Several measures assess common mental disorders (CMD) including depression, anxiety and somatic symptoms using sub-scales within one instrument. This analysis emphasizes the measurement of depression symptoms, but as symptoms of CMD often co-occur, attention is also given to the role that other symptoms might play in the Vietnamese experience of depression. Several studies validate or use more than one measure of depression. A list of the measures and their characteristics is found in Table 1.

\section{Results}

\section{Population}

The studies reviewed measures used in Vietnam and among populations of Vietnamese living abroad. In several cases, different studies present data from the same study sample, while some present data from more than one study site or sample. Of the studies undertaken in Vietnam, seven present data from the north [24-30], five from the central provinces $[6,14,24,27,31]$, and eight from the south $[6,15,24,27,31-35]$. In addition to studies conducted among general adult populations, two studies focused on older adults [35, 36], one on youth [14], five on mothers [24, 25, 27, 30, 37], one on men [28] and one on 'Amerasians', who are identified as a vulnerable population due to discrimination [38]. Of the studies taking place outside of Vietnam, one was in Taiwan among Vietnamese women [39], two were in Australia among adult immigrants and refugees [34, 40], and eight were in the United States (US), mostly with refugees or immigrants [38, 41-47]. One study identified the study population as Vietnamese Americans [47].

\section{Validation}

The detailed results of the review are presented in Table 2. Of the ten measures found, eight were explicitly validated, including the: CES-D, CIDI, DASS, GHQ, iHSCL, PVPS, SRQ-20 and the VDS. Four of the measures were validated by one study each, while the iHSCL was validated by three studies (all in the US), the PVPS was validated by two (one in Australia and one in south Vietnam), the SRQ-20 was validated by three studies (in north, central and south Vietnam), and the VDS was validated by four (three in the US, one in south Vietnam). Of 


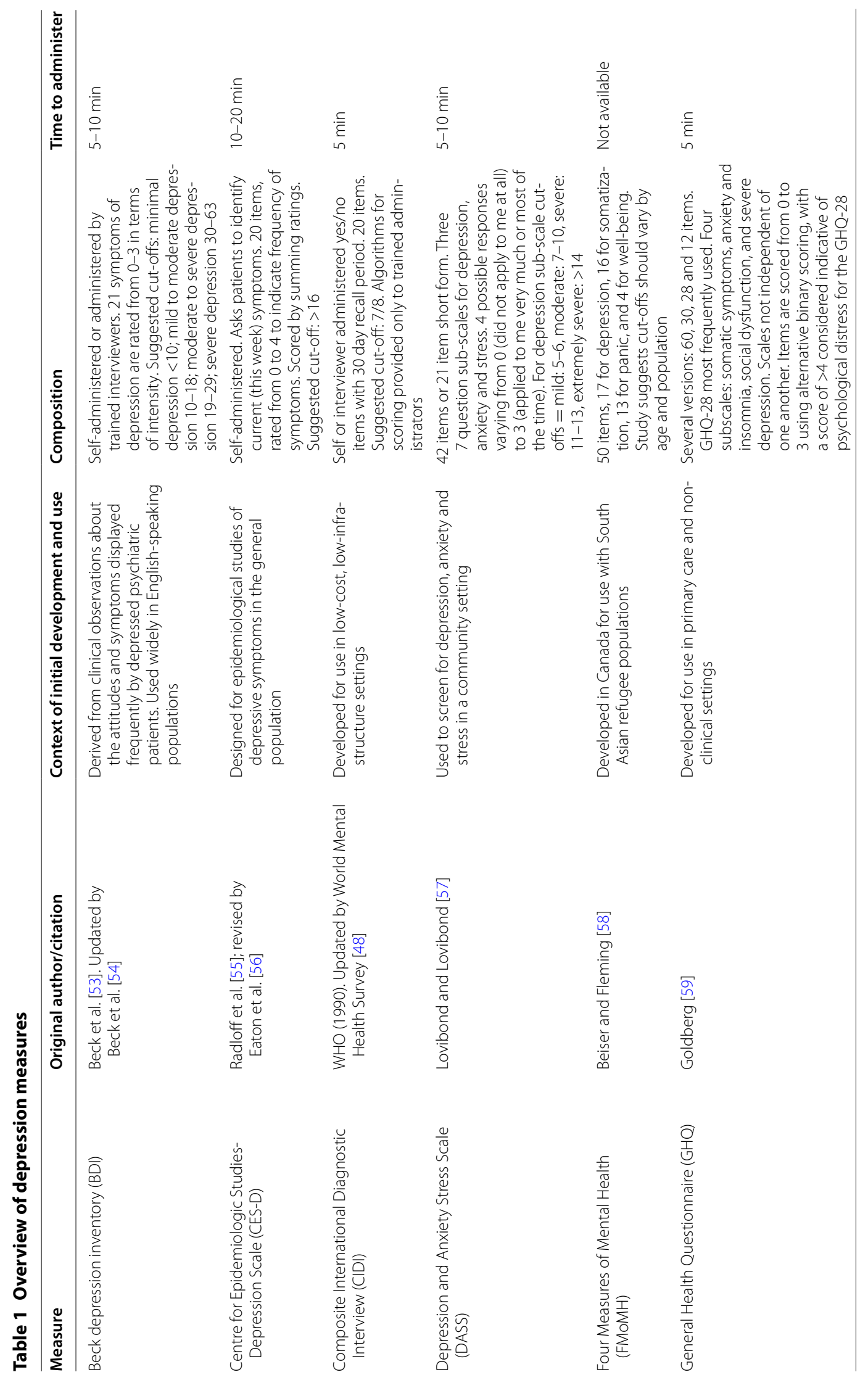




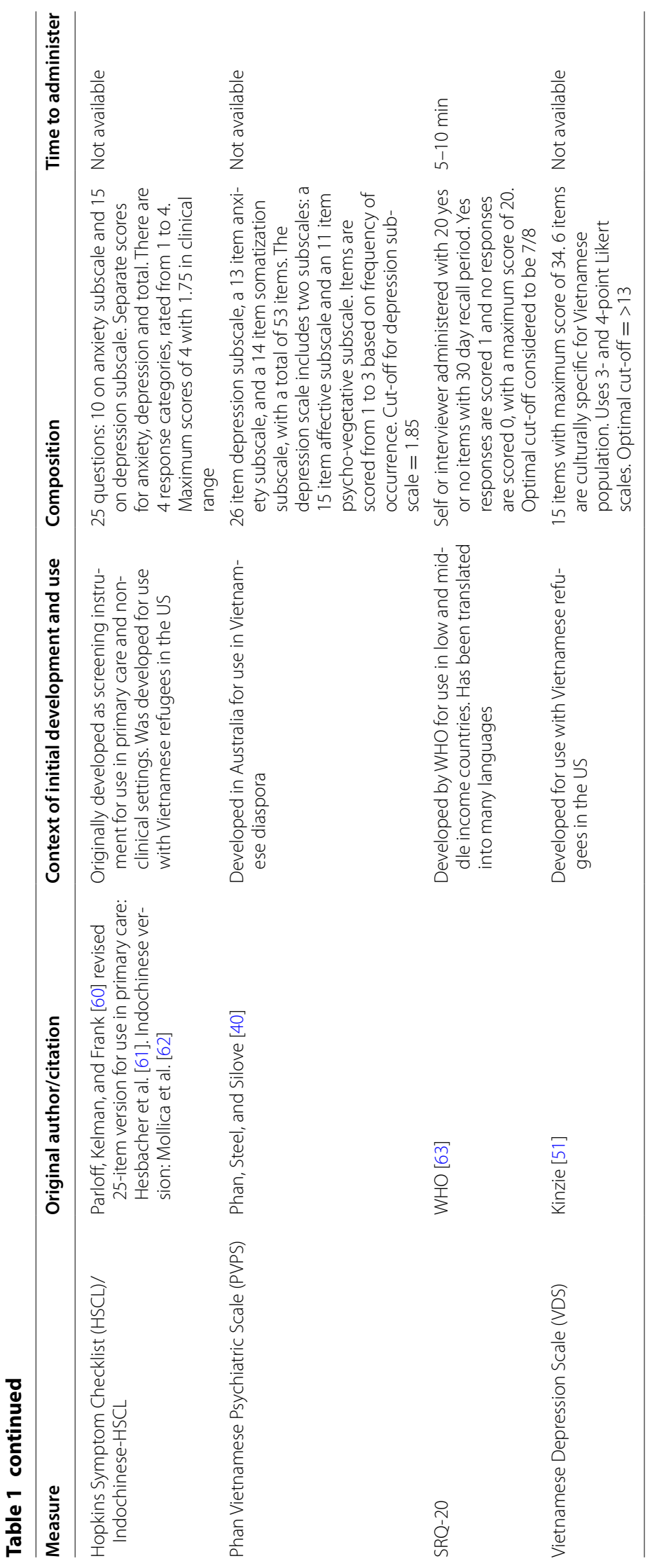


the validated measures, only five were validated for content validity (CES-D, CIDI, iHSCL, PVPS, SRQ-20), while seven were validated for construct validity (CIDI, DASS, GHQ, iHSCL, PVPS, SRQ-20, and VDS). Six measures were assessed for criterion validity (CIDI, DASS, iHSCL, PVPS, SRQ-20, and VDS) and four were tested for reliability (CES-D, DASS, PVPS, SRQ-20). The results of content, construct and criterion validity and reliability testing as assessed by each study are described in Table 2 .

\section{Characteristics of measures}

While most studies did not assess the acceptability of the measures for use in primary care, the characteristics of the measures, including their brevity and accessibility, can help to inform decisions about their use in such settings. Of the validated measures, four were explicitly developed for use in non-clinical or community settings (DASS, GHQ, iHSCL and SRQ-20). The CES-D was developed for use in epidemiological studies, while the CIDI requires certification by the WHO-WMH, but training is unavailable in Vietnamese [48]. The PVPS is 53 items long, making it lengthy for use in time-sensitive settings. In the US contexts in which it was used, the iHSCL is described as easy to use, with clear language, and acceptable to target populations [43, 46] Based on its use in Vietnam, the brevity of the SRQ-20, the ease of administration and training, and cost-effectiveness are described as benefits of the measure [6,31].

\section{Discussion}

While there are numerous methods of assessing validity, definitions of "validation" and its associated methodology vary [20]. For the purposes of this review, we have taken into account the following criteria when assessing the extent to which validity of measures for use in primary care among Vietnamese populations has been tested: the extent to which a measure has been validated, including testing for construct and content validity in addition to criterion validity and reliability; the populations in which measures were tested, including the diversity of the populations and whether the measures were tested in Vietnam or among the diaspora; the length of the measures and ease of use.

While the review identified 11 studies that explicitly validated at least one measure of depression, the methods used and criteria on which conclusions were drawn were variable. While some studies reported minimally on content validity and reliability [47], others applied extensive testing [40]. The potential variation in cultural meaning and experience of depression in Vietnamese populations is an important issue that requires further study [40, 41, 49]. Consequently, testing only criterion validity and reliability does not assess whether depression as defined by the measure appropriately captures the culture-specific construct of depression. In studies that validate Western-developed measures for use in Vietnamese populations, assessing content and construct validity are critical aspects in determining if a measure is in fact valid. Many studies in this review examined the validity of measures in terms of their ability to determine caseness, but did not test the construct validity of the measure. Stratton et al.s [6] psychometric evaluation of the Self Reporting Questionnaire-20 (SRQ-20) was a response to this gap in the literature. In addition, when criterion validity was assessed it was often based on comparison with international psychiatric diagnostic assessments $[30,40,45]$ that have not been validated for use in Vietnam. The absence of somatic symptoms in these measures was associated with underreporting of depression in Vietnamese subjects $[33,50]$. Assessing validity based on comparisons with these measures may be problematic.

The review included two measures that were developed for use among Vietnamese populations [40,51] and one that was adapted for use among South Asian populations $[41,50]$, raising the question of whether indigenously derived measures are superior to adapted Western measures. The findings of this review suggest that a balance of culture-specific and universal constructs is essential for identifying depression in Vietnamese populations [41, 50]. Whether this can be accomplished sufficiently by modifying Western measures is debatable. In their work on depression in Zimbabwe, Patel et al. [52] found that Western derived measures could be used cross-culturally with sufficient attention to the translation of culture-specific concepts. All instruments would benefit from extensive evaluation of content and construct validity prior to being used in new populations.

This review includes studies that took place in Vietnam, or which took place among the Vietnamese diaspora. There are sizeable Vietnamese populations living in many countries, whose experience as migrants distinguishes them from Vietnamese living in Vietnam. The studies included in this review focused almost exclusively on immigrant or refugee populations and did not explicitly validate the measures for use among populations of Vietnamese descent living in these countries. While Vietnamese diaspora populations would certainly benefit from culturally appropriate measures of depression in primary care, consideration of the diversity of experience among these populations should be made when selecting appropriate measures. Vietnam itself is a diverse country with linguistic and cultural differences between regions, among rural and urban populations and among the diverse minority ethnic populations. For this reason, the validation of measures within specific populations should be considered before use. Many measures were only 


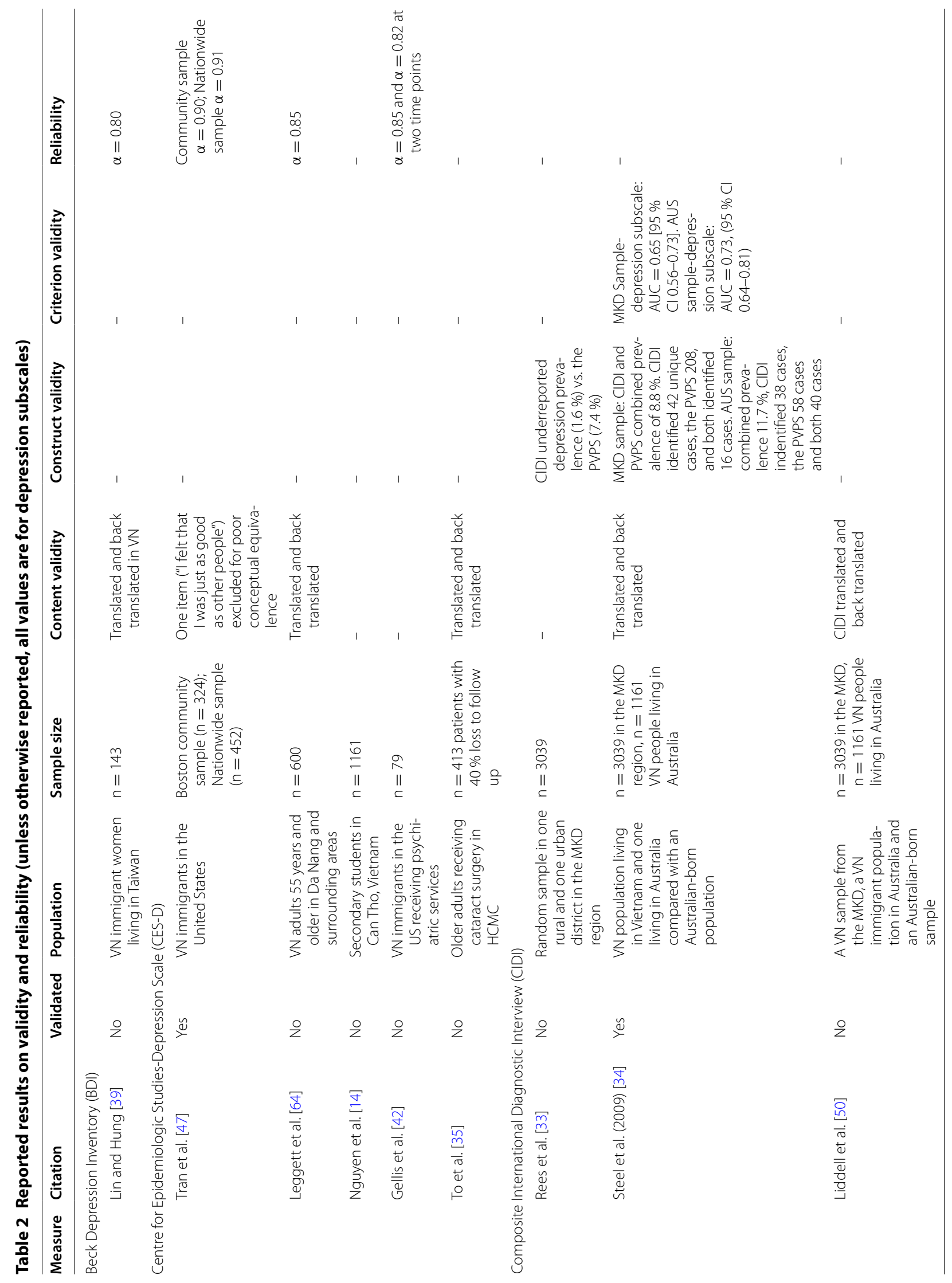




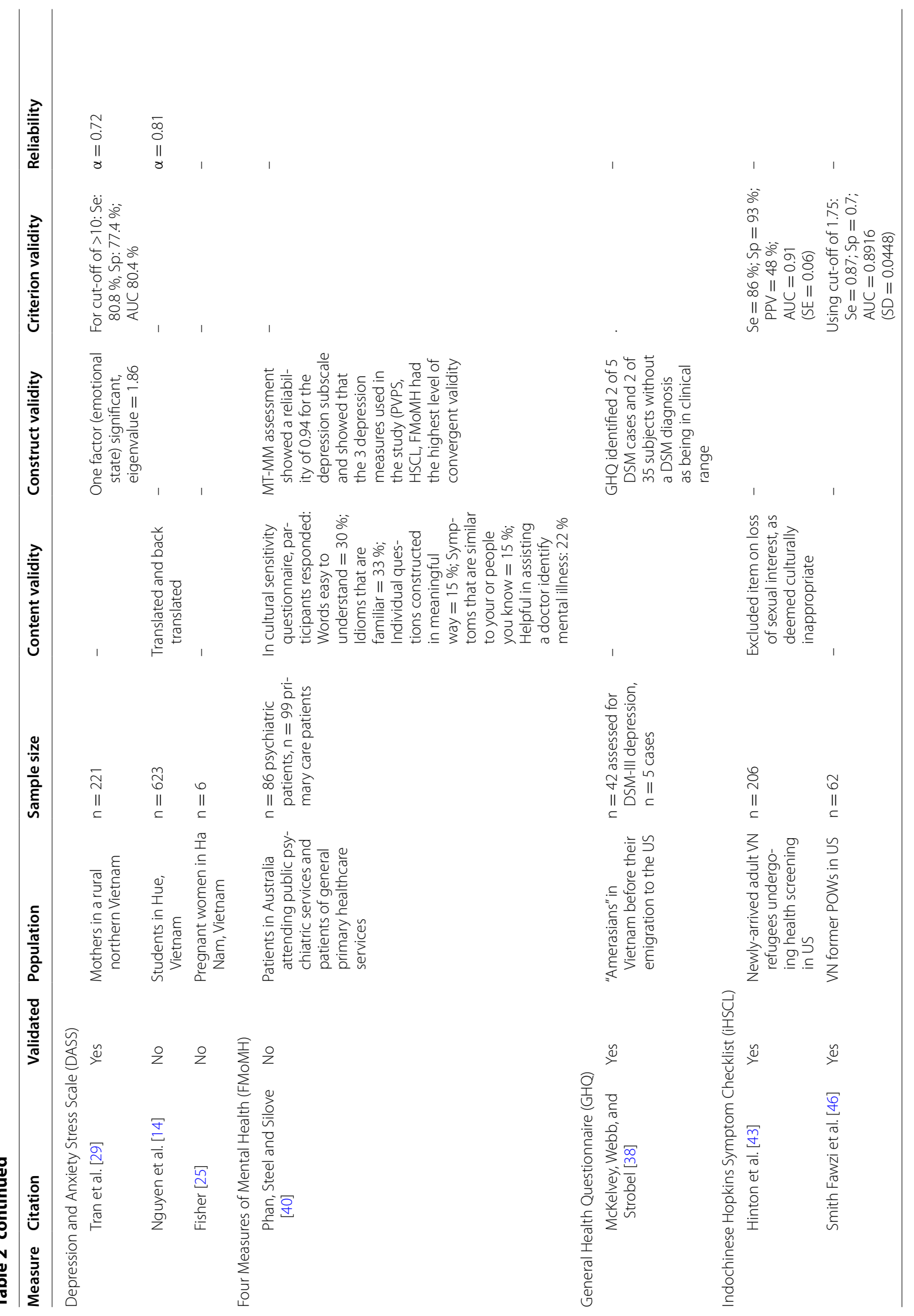









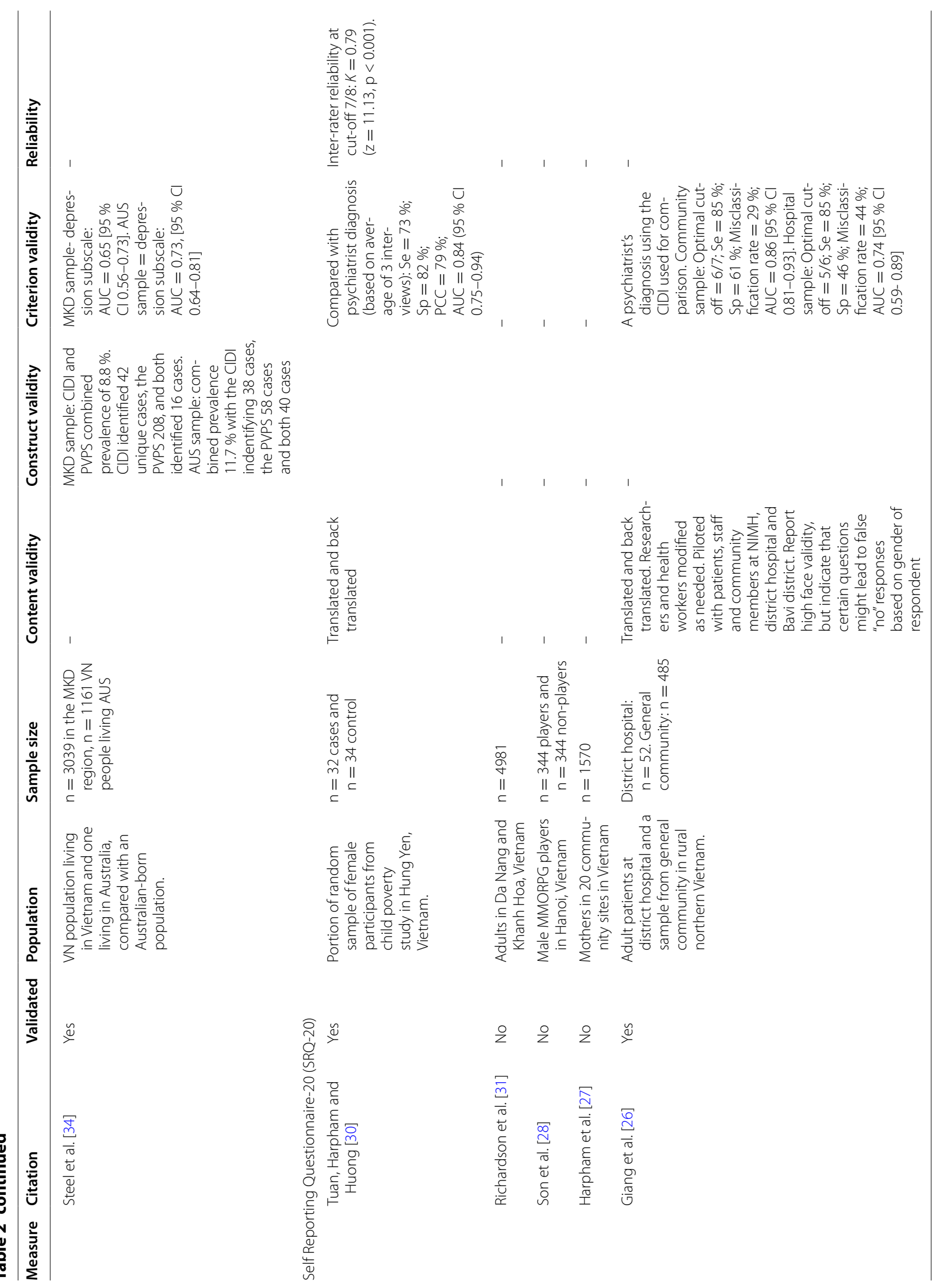




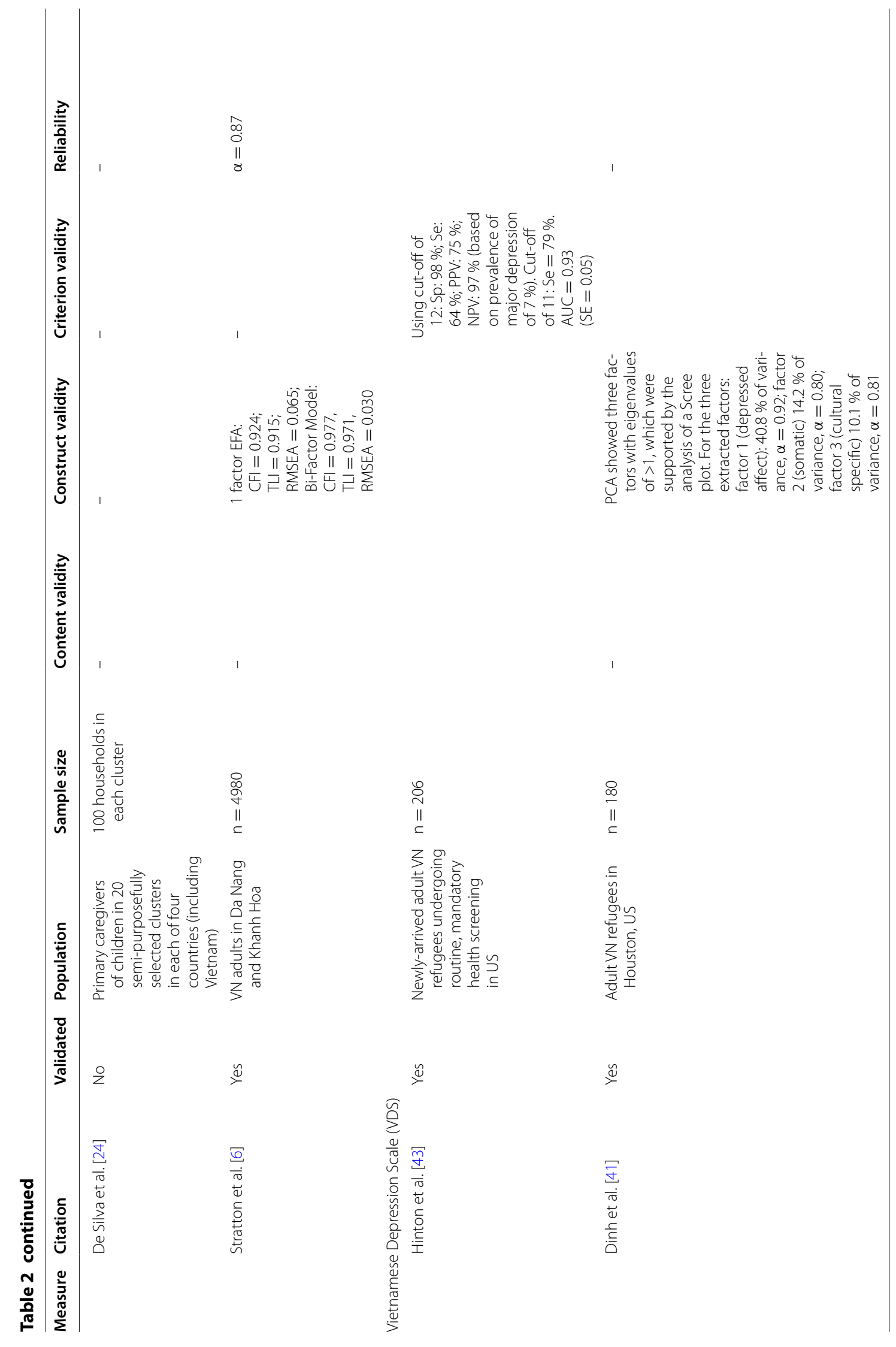




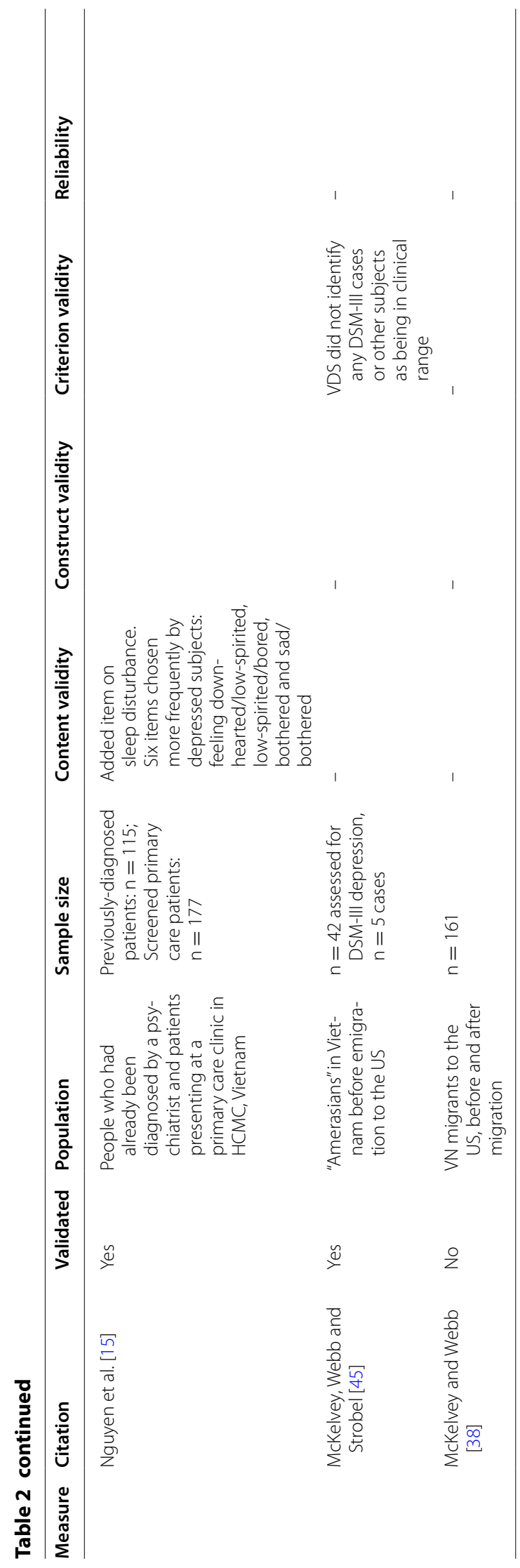


validated in one region of Vietnam or among one population sub-group. This should be considered when selecting measures for use in the country. Notably, no studies validated measures among minority population groups. This is a significant gap and additional research to validate measures for use in these populations is required.

Special considerations are also required for the use of depression measures in primary care environments, which often involve time and resource constraints and rely on non-specialist health staff. Measures must be brief, easy to use and to interpret, cost-effective and accessible. The PVPS, while extensively validated and based on a culturally relevant construct of CMD, is 53-items long with a 26-item depression subscale, making it lengthy for use in primary care environments.

Drawing definitive conclusions regarding the most appropriate measures for use among Vietnamese populations is difficult. The iHSCL may be most appropriate for use among immigrant or refugee populations. It includes 25 items, with a 15 -item depression subscale, and has been found to be easy to use, with clear language. While it was found acceptable by refugees living in the US it has been only minimally tested in Vietnam and should be further validated for use in the country and for use among more established Vietnamese communities. The SRQ-20, which was validated and used in several regions of Vietnam, was found to be brief, easy for training and use and cost-effective [6], and thus appears most suitable for use in primary care settings in Vietnam. There are, however, concerns about differences between men and women when responding to the measure $[6,26]$ and it has not been validated among minority populations. Further research about differences in validity between urban and rural populations is also recommended.

\section{Limitations}

This review includes only studies published in English. While we intended to include Vietnamese journals in this review, we found that as these journals are not indexed it was not feasible to include them. Further research in this area would benefit from including research published in Vietnamese journals. It was not possible to conduct a quantitative comparison across measures. The results therefore describe statistical results as reported in the studies and qualitative assessments.

\section{Conclusions}

Assessing the validity of depression measures, their cultural acceptability and their appropriateness for use in primary care requires a balance of standard validity testing, assessment within the specific sociocultural context, and criteria such as brevity and cost-effectiveness.
Vietnam is not only ethnically diverse but also has linguistic and cultural diversity by region that mean that, even when found to be valid in one population or region, a measure might require additional testing for use elsewhere. The increasing influence of Western culture, particularly in urban areas, may also lead to variation among the validity of measures between rural and urban populations. As demonstrated through testing of the SRQ20, differences in gender in Vietnam may also mean that measures that can be used universally in Western populations will require gender-specific assessment in Vietnam.

The variability in methods used to validate instruments and populations in which the measures were tested makes definitive conclusions about the most effective measure difficult. Studies or services wishing to implement depression screening in primary care have an extensive body of literature on which to draw to make decisions about the most appropriate instrument for their purposes. Specific consideration, however, to population, region and gender specific factors should be given when using these measures in practice. Pilot testing of measures validated in one region or population to be used in another is recommended in order to ensure conceptual and linguistic equivalence of measures across the diverse Vietnamese population.

While a discussion of the validation of depression measures in other contexts was beyond the scope of this review, the findings have implications beyond Vietnamese populations. We found little consistency in the validation of depression measures, with limited attention to construct and content validity, both of which are important to determine cross-cultural validity. To guide further studies of validation of psychometric measures in cross-cultural contexts and in primary care, enhanced methodological guidelines for validation, which include testing of all types of validity and which draw on anthropological methods to avoid pitfalls of testing against nonvalidated Western psychiatric assessments, would benefit researchers conducting validation studies and health services wishing to employ the most appropriate measure.

\section{Abbreviations \\ Depression measures \\ BDI: Beck Depression Inventory; CES-D: Centre for Epi- demiologic Studies-Depression Scale; CIDI: Composite International Diagnostic Interview; DASS: Depression and Anxiety Stress Scale; FMoMH: Four Measures of Mental Health; GHQ: General Health Questionnaire; HSCL: Hopkins Symptom Checklist; iHSCL: Indochi- nese Hopkins Symptom Checklist; PVPS: Phan Viet- namese Psychiatric Scale; SRQ-20: Self-Reporting Questionnaire-20; VDS: Vietnamese Depression Scale}




\section{Statistical tests}

Se: sensitivity; Sp: specificity; AUC: area under the receiver operating curve; PPV: positive predictive value; NPV: negative predictive value; PCC: probability of correct classification; $K$ : kappa coefficient; EFA: exploratory factor analysis; CFI: Comparative Fit Index; TLI: TuckerLewis Index; RMSEA: root mean square error of approximation; PCA: principal component analysis; MT-MM: multitrait-multimeasure

\section{Contextual acronyms}

NIMH: National Institute of Mental Health; HCMC: Ho Chi Minh City; VN: Vietnamese; MMORPG: Mix multiplayer online role playing game; MKD: Mekong Delta Region; IDRC: International Development Research Centre; DSM: Diagnostic and Statistical Manual; US: United States; WHO: World Health Organization; POW: Prisoner of War; DIS: Diagnostic Interview Schedule for DSM

\section{Authors' contributions}

JM coordinated and executed the review, conducted the analysis and drafted the manuscript. EG conceived the research question, advised on the review process and participated in drafting the manuscript. CG provided extensive feedback on the results of the review and on the draft of the manuscript. PTO and WZ assisted with the review and analysis. KC helped to conceptualize the paper and provided guidance on the draft. VCN provided guidance on the analysis and draft. All authors read and approved the final manuscript.

\section{Author details}

1 Faculty of Health Sciences, Simon Fraser University, Blusson Hall, Room 11300, 8888 University Drive, Burnaby, BC V5A 1S6, Canada. ${ }^{2}$ Arthritis Research Centre of Canada, 5591 No. 3 Rd., Richmond, BC V6X 2C7, Canada. ${ }^{3}$ Institute of Population, Health and Development (PHAD), Alley No. 18, 132 Hoa Bang St., Cau Giay, Hanoi, Vietnam. ${ }^{4}$ Department of Psychological and Brain Sciences, Dartmouth College, 6207 Moore Hall, Hanover, NH 03755, USA. ${ }^{5}$ School of Public Health and Health Systems, University of Waterloo, Waterloo, ON N2L 3G1, Canada.

\section{Acknowledgements}

This work was funded by Grand Challenges Canada [Grant 0330-04]; the first author was provided doctoral funding by the International Development Research Centre Doctoral Research Award.

\section{Compliance with ethical guidelines}

\section{Competing interests}

The authors declare that have no competing interests.

Received: 1 May 2015 Accepted: 12 August 2015

Published online: 19 August 2015

\section{References}

1. Murray CJL, Vos T, Lozano R, Naghavi M, Flaxman AD, Michaud C, et al. Disability-adjusted life years (DALYs) for 291 diseases and injuries in 21 regions, 1990-2010: a systematic analysis for the Global Burden of Disease Study 2010. The Lancet. 2012;380(9859):2197-223.

2. Jenkins JH, Kleinman A, Good BJ. Cross-cultural aspects of depression. In: Becker J, Kleinman A, editors. Advances in affective disorders: theory and research, Vol I, psychosocial aspects. Erlbaum Press; 1991. p. 67-99.
3. Patel V, Thornicroft G. Packages of care for mental, neurological, and substance use disorders in low- and middle-income countries: PLoS Medicine Series. PLoS Med. 2009;6(10):e1000160. doi:10.1371/journal. pmed.1000160.

4. mhGap. Scaling Up care for mental, neurological and substance use disorders. Geneva: World Health Organization, 2008. Contract no.: report.

5. World Health Organization. Integrating mental health services into primary health care: mental health policy, planning and services development Geneva: World Health Organization; 2007. Contract No.: Report.

6. Stratton KJ, Aggen SH, Richardson LK, Acierno R, Kilpatrick DG, Gaboury MT, et al. Evaluation of the psychometric properties of the Self-Reporting Questionnaire (SRQ-20) in a sample of Vietnamese adults. Compr Psychiatry. 2013;54(4):398-405.

7. The World Bank. Vietnam 2014 [cited 2014 July 12]. http://www.worldbank.org/en/country/vietnam. Accessed 12 Jul 2014.

8. Affairs IWGfl. Indigenous peoples in Vietnam N.D. [cited 2014 July 12]. http://www.iwgia.org/regions/asia/vietnam. Accessed 12 Jul 2014.

9. Migration IOf. No Title 2012 [cited 2014 July 12]. http://www.iom.int.vn/ joomla/index.php?option=com_content\&task=view\&id=346\&ltemid= 1. Accessed 12 Jul 2014

10. Niemi EM, Falkenberg T, Nguyen MTT, Nguyen MTN, Patel V, et al. The social contexts of depression during motherhood: a study of explanatory models in Vietnam. J Affect Disord. 2010;124(1-2):29-37.

11. Vuong DA, Van Ginneken E, Morris J, Ha ST, Busse R. Mental health in Vietnam: burden of disease and availability of services. Asian J Psychiatry. 2011;4(1):65-70.

12. World Health Organization-AIMS. WHO-AIMS report on mental health system in Vietnam. Hanoi: WHO and Ministry of Health, Vietnam. Hanoi, Vietnam World Health Organization and Ministry of Health, Vietnam 2006.

13. $\mathrm{Ng} \mathrm{CH}$, Than PT, La CD, Van Than Q, Van Dieu C. The national community mental health care project in Vietnam: a review for future guidance. Australas Psychiatry. 2011;19(2):143-50.

14. Nguyen D, Dedding C, Pham T, Wright P, Bunders J. Depression, anxiety, and suicidal ideation among Vietnamese secondary school students and proposed solutions: a cross-sectional study. BMC Public Health. 2013;13(1):1195.

15. Nguyen N-LD, Hunt DD, Scott CS. Screening for depression in a primary care setting in Vietnam. J Nerv Mental Dis. 2005;193(2):144-7.

16. Niemi M, Thanh HT, Tuan T, Falkenberg T. Mental health priorities in Vietnam: a mixed-methods analysis. BMC Health Ser Res. 2010;10:257.

17. Arksey H, O'Malley L. Scoping studies: towards a methodological framework. Int J Soc Res Methodol. 2005;8(10):19-32.

18. Niemi M, Målqvist M, Giang KB, Allebeck P, Falkenberg P. A narrative review of factors influencing detection and treatment of depression in Vietnam. Int J Mental Health Syst. 2013;7:15. doi:10.1186/1752-4458-7-15.

19. Wittig U, Lindert J, Merbach M, Brähler E. Mental health of patients from different cultures in Germany. Eur Psychiatry. 2008;23:S28-35.

20. Cook DA, Beckman TJ. Current concepts in validity and reliability for psychometric instruments: theory and application. Am J Med. 2006;119(2):166e7.

21. DeVon HA, Block ME, Moyle-Wright P, Ernst DM, Hayden SJ, Lazzara DJ, et al. A psychometric toolbox for testing validity and reliability. J Nurs Scholarsh. 2007;39(2):155-64.

22. Flaherty JA, Gaviria FM, Pathak D, Mitchell T, Wintrob R, Richman JA, et al Developing instruments for cross-cultural psychiatric research. J Nerv Ment Dis. 1988;176(5):257-63.

23. Guillemin F, Bombardier C, Beaton D. Cross-cultural adaptation of health-related quality of life measures: literature review and proposed guidelines. J Clin Epidemiol. 1993;46(12):1417-32.

24. De Silva MJ, Huttlya SR, Harpham T, Kenward MG. Social capital and mental health: a comparative analysis of four low income countries. Soc Sci Med. 2007;64(1):5-20.

25. Fisher J, Hau N, Mannava P, Ha T, Thao D, Huong T, et al. Translation, cultural adaptation and field-testing of the Thinking Healthy Program for Vietnam. Glob Health. 2014;10:37.

26. Giang KB, Allebeck P, Kullgren G, Van Tuan N. The Vietnamese Version of the Self Reporting Questionnaire 20 (SRQ-20) in detecting mental disorders in rural Vietnam: a validation study. Int J Soc Psychiatry. 2006:52(2):175-84.

27. Harpham T, Huttly S, De Silva MJ, Abramsky T. Maternal mental health and child nutritional status in four developing countries. J Epidemiol Community Health. 2005;59(12):1060-4. 
28. Son DT, Yasuoka J, Poudel KC, Otsuka K, Jimba M. Massively multiplayer online role-playing games (MMORPG): association between its addiction, self-control and mental disorders among young people in Vietnam. Int J Soc Psychiatry. 2013;59(6):570-7.

29. Tran TD, Biggs B-A, Tran T, Casey GJ, Hanieh S, Simpson JA, et al. Psychological and social factors associated with late pregnancy iron deficiency anaemia in rural Viet Nam: A population-based prospective study. PLoS One. 2013;8(10):e78162.

30. Tuan T, Harpham T, Huong NT. Validity and reliability of the Selfreporting Questionnaire 20 items in Vietnam. Hong Kong J Psychiatry. 2004;14(3):15-8.

31. Richardson LK, Amstadter AB, Kilpatrick DG, Gaboury MT, Tran TL, Trung LT, et al. Estimating mental distress in Vietnam: the use of the SRQ-20. Int J Soc Psychiatry. 2010;56(2):133-42.

32. Thuy N, Berry H. Social capital and mental health among mothers in Vietnam who have children with disabilities. Global Health Action. 2013;6. doi:10.3402/gha.v6i0.18886.

33. Rees S, Silove D, Chey T, Steel Z, Bauman A, Phan T. Physical activity and psychological distress amongst Vietnamese living in the Mekong Delta. Aust N Z J Psychiatry. 2012;46(10):966-71.

34. Steel Z, Silove D, Giao NM, Phan TTB, Chey T, Whelan A, et al. International and indigenous diagnoses of mental disorder among Vietnamese living in Vietnam and Australia. Br J Psychiatry. 2009;194(4):326-33.

35. To KG, Meuleners LB, Fraser ML, Van Duong D, Van Do D, Huynh V-AN, et al. The impact of cataract surgery on depressive symptoms for bilateral cataract patients in Ho Chi Minh City, Vietnam. Int Psychogeriatr. 2014;26(2):307-13.

36. Leggett A, Zarit SH, Hoang CN, Nguyen HT. Correlates of cognitive impairment in older Vietnamese. Aging Mental Health. 2013;17(8):915-23.

37. Tran TD, Tran T, La B, Lee D, Rosenthal D, Fisher J. Screening for perinatal common mental disorders in women in the north of Vietnam: a comparison of three psychometric instruments. J Affect Disord. 2011;133(1-2):281-93

38. McKelvey RS, Webb JA. Premigratory expectations and postmigratory mental health symptoms in Vietnamese Amerasians. J Am Acad Child Adolesc Psychiatry. 1996;35(2):240-5.

39. Li-Hua L, Chich-Hsiu H. Vietnamese women immigrants'life adaptation, social support, and depression. J Nurs Res (Taiwan Nurses Association). 2007;15(4):243-54.

40. Phan T, Steel Z, Silove D. An ethnographically derived measure of anxiety, depression and somatization: the phan Vietnamese psychiatric scale. Transcult Psychiatry. 2004;41(2):200-32.

41. Dinh TQ, Yamada AM, Yee BWK. A culturally relevant conceptualization of depression: an empirical examination of the factorial structure of the Vietnamese depression scale. Int J Soc Psychiatry. 2009;55(6):496-505.

42. Gellis ZD. Kin and Nonkin Social supports in a community sample of Vietnamese immigrants. Soc Work. 2003;48(2):248-58.

43. Hinton WL, Du N, Chen YC, Tran CG, Newman TB, Lu FG. Screening for major depression in Vietnamese refugees: a validation and comparison of two instruments in a health screening population. J Gen Intern Med. 1994;9(4):202-6.

44. McKelvey RS, Webb JA. Comparative levels of psychological distress in a pre-migratory refugee population. Aust N Z J Psychiatry. 1997;31(4):543-8.

45. McKelvey RS, Webb JA, Strobel RM. The prevalence of psychiatric disorders among Vietnamese Amerasians: a pilot study. Am J Orthopsychiatry. 1996;66(3):409-15.

46. Smith Fawzi MC, Murphy E, Pham T, Lin L, Poole C, Mollica RF. The validity of screening for post-traumatic stress disorder and major depression among Vietnamese former political prisoners. Acta Psychiatri Scand. 1997;95(2):87-93.

47. Tran TV, Ngo D, Conway K. A cross-cultural measure of depressive symptoms among Vietnamese Americans. Social Work Res. 2003;27(1):56-64.

48. World Health Organization. The World Health Organization world menta health composite international diagnostic interview (WHO WMH-CIDI): World Health Organization 2004 [cited 2014 July 12, 2014]. http://www. hcp.med.harvard.edu/wmhcidi/. Accessed 12 Jul 2014.

49. Fry AJ, Nguyen T. Culture and the self: implications for the perception of depression by Australian and Vietnamese nursing students. J Adv Nurs. 1996;23(6):1147-54
50. Liddell BJ, Chey T, Silove D, Phan TTB, Giao NM, Steel Z. Patterns of risk for anxiety-depression amongst Vietnamese-immigrants: a comparison with source and host populations. BMC Psychiatry. 2013;13(1):1-22.

51. Kinzie JD, Manson SM, Tolan NT, Anh B, Pho TN. Development and validation of a Vietnamese-language depression rating scale. Am J Psychiatry. 1982;139(10):1276-8.

52. Patel V, Abas M, Broadhead J, Todd C, Reeler A. Depression in developing countries: lessons from Zimbabwe. BMJ Br Med J. 2001;322(7284):482-4.

53. Beck AT, Ward CH, Mendelson M, Mock J, Erbaugh J. An inventory for measuring depression. Arch Gen Psychiatry. 1961;4:561-71.

54. Beck AT, Steer RA, Ball R, Ranieri WF. Comparison of beck depression inventories-IA and -II in psychiatric outpatients. J Pers Assess. 1996;67(3):588

55. Radloff LS. The CES-D scale: a self-report depression scale for research in the general population. Appl Psychol Meas. 1977;1(3):385-401.

56. Eaton WW, Muntaner C, Smith C, Tien A, Ybarra M. Center for epidemiologic studies depression scale: review and revision (CESD and CESD-R). In: MME, editor. The use of psychological testing for treatment planning and outcomes assessment. 3rd ed. Mahwah, NJ: Lawrence Erlbaum; 2004. P 363-77.

57. Lovibond SHL, Lovibond PF. Manual for the depression anxiety stress scales. Sydney: Psychology Foundation; 1995.

58. Beiser M, Fleming JAE. Measuring psychiatric disorder among Southeast Asian refugees. Psychol Med. 1986;16(03):627-39.

59. Goldberg D. The detection of psychiatric illness by questionnaire: a technique for the identification and assessment of non-psychotic psychiatric illness. London: Oxford University Press; 1972.

60. Parloff MB, Kelman HC, Frank JD. Comfort, effectiveness, and selfawareness as criteria for improvement in psychotherapy. Am J Psychiatry. 1954;3:343-51.

61. Hesbacher PT, Rickels K, Morris RJ, Newman H, Rosenfeld H. Psychiatric illness in family practice. J Clin Psychiatry. 1980;41(1):6-10.

62. Mollica RF, Wyshak G, Marneffe DE, Khuon F, Lavelle J. Indochinese versions of the Hopkins Symptom Checklist- 25: a screening tool for the psychiatric care of refugees. Am J Psychiatry. 1987;144(4):497-500.

63. World Health Organization. A user's guide to the Self-Reporting Questionnaire. 1994 [cited 2014]. July 12]. Available from: http://whqlibdoc. who.int/hq/1994/WHO_MNH_PSF_94.8.pdf. Accessed 12 Jul 2014

64. Leggett A, Zarit SH, Nguyen NH, Hoang CN, Nguyen HT. The influence of social factors and health on depressive symptoms and worry: a study of older Vietnamese adults. Aging Mental Health. 2012;16(6):780-6.

\section{Submit your next manuscript to BioMed Central and take full advantage of:}

- Convenient online submission

- Thorough peer review

- No space constraints or color figure charges

- Immediate publication on acceptance

- Inclusion in PubMed, CAS, Scopus and Google Scholar

- Research which is freely available for redistribution

Submit your manuscript at

www.biomedcentral.com/submit
() Biomed Central 